\title{
POLARIZED POSITRON PRODUCTION AND TRACKING AT THE ILC POSITRON SOURCE*
}

\author{
A. Ushakov ${ }^{\dagger}$, S. Riemann, A. Schälicke, DESY, Germany
}

\section{Abstract}

The positron source planned for the future International Linear Collider (ILC) is based on a helical undulator system. Depending on the accelerator design it will be possible to get polarized positrons at the interaction point. A source performance with high positron yield and high polarization is the aim of our design studies. We focus on the optimization of target and capture section by combining the advantages of the simulation codes FLUKA, Geant 4 and ASTRA.

\section{INTRODUCTION}

The operation with polarized electron and positron beams will significantly extend the physics potential of the future ILC. Already a positron polarisation of approximately $30 \%$ will improve the precision of measurements substantially.

In this work the polarization of the positron beam produced by the undulator based positron source [1] with Tiand $\mathrm{W}$-alloy targets is examined using Geant 4 . The new release of Geant 4 includes the spin dependence of all QED processes and allows to perform a helicity-dependent tracking of particles through target and capture section. The ASTRA code is used to calculate the positron capture efficiency into the optical matching device and preaccelerator. Parameters as positron yield, capture efficiency and deposited energy obtained from Geant 4 and FLUKA simulations are compared; activation aspects have been considered in earlier FLUKA studies [2].

\section{POSITRON PRODUCTION}

The undulator based system uses a helical undulator placed at the $150 \mathrm{GeV}$ point of the ILC electron linac. The electron beam passing through the undulator generates circularly polarized photons [3]. The energy distribution and polarization of the photons radiated in helical undulator with period of $1.15 \mathrm{~cm}$ and undulator $K$ value of 0.92 are shown in Fig. 1. Each electron passing one meter of the undulator produces 1.94 photons.

The distance between end of undulator and target is 500 meters. No photon collimator has been placed between the undulator and the target. The rms photon beam spot size on the target is $3.9 \mathrm{~mm}$.

\footnotetext{
* Work supported by the Commission of the European Communities under the 6th Framework Programme "Structuring the European Research Area", contract number RIDS-011899.

$\dagger$ andriy.ushakov@desy.de
}

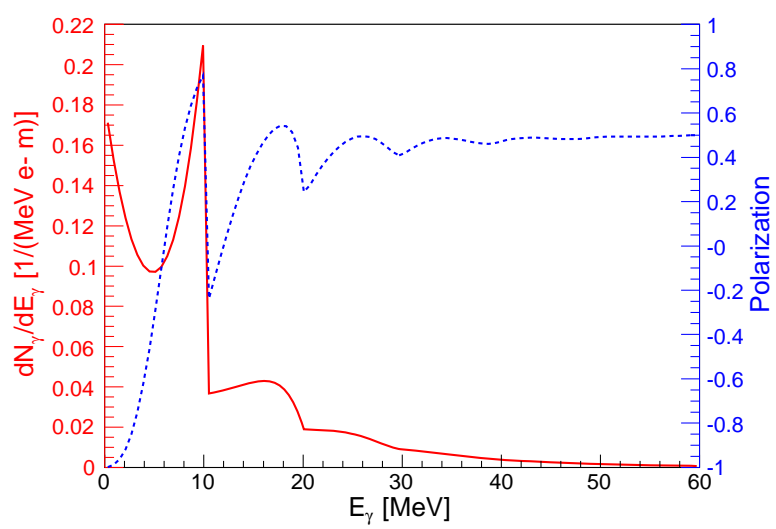

Figure 1: Energy distribution and polarization of photons generated by an electron passing one meter of the undulator (period is $1.15 \mathrm{~cm}$ and $K$ value is 0.92 ).

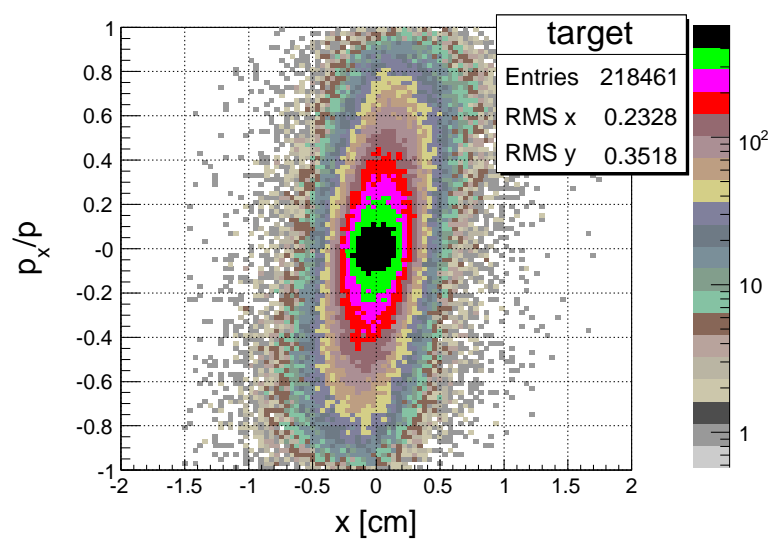

Figure 2: The positron $x x^{\prime}$ phase space after the target calculated by FLUKA for $10^{7}$ primary photons.

The photons hit a thin Ti6Al4V alloy target $(6 \%$ of $\mathrm{Al}$ and $4 \%$ of $\mathrm{V}$ ) of 0.4 radiation length and produce electronpositron pairs.

The positron production in the target has been calculated with FLUKA [4] and Geant4 [5]. The positron $x x^{\prime}$ phase space after the target calculated by FLUKA for $10^{7}$ primary photons is shown in Fig. 2. According to FLUKA simulation, the positron yield defined as number positrons per incident on the Ti6Al4V target photon is $2.18 \cdot 10^{-2}$. The positron yield of $2.19 \cdot 10^{-2}$ calculated with Geant 4 is in very good agreement with the FLUKA result.

The density distribution of the power deposited in the target by the $117 \mathrm{~kW}$ photon beam is shown in Fig. 3. The average energy deposited in the target by one photon is $847.7 \mathrm{keV}$ (FLUKA) and $854 \mathrm{keV}$ (Geant4) correspond- 
ing to $8.14 \%$ (FLUKA) or $8.2 \%$ (Geant 4 ) of photon mean energy.

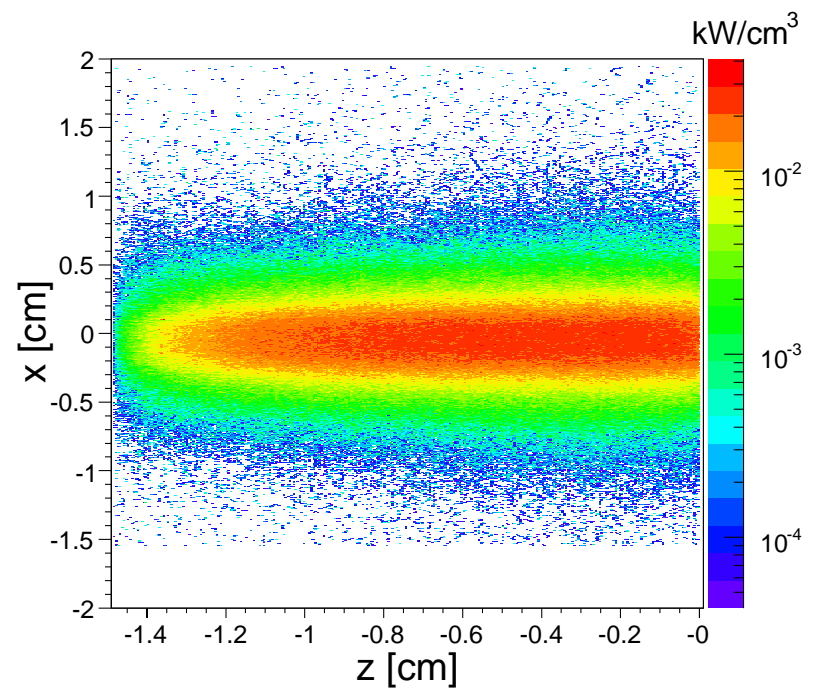

Figure 3: The power deposited in the target by $117 \mathrm{~kW}$ photon beam (FLUKA).

It should be remarked that it is not yet possible to implement electric fields into particle tracking simulations with FLUKA. Hence, ASTRA and Geant 4 were used to model the capture section for tracking. In addition, with Geant4 the positron beam polarization can be determined. The average longitudinal polarization of positrons escaping the target is $27 \%$. The positron energy distribution and the polarization of the positrons immediately after the target are shown in Figs. 4 and 5.

The simulations were also performed for the same photon beam hitting a W25Re target. A tungsten or tungsten alloy target has a high positron yield and is therefore also considered as possible material for the target.

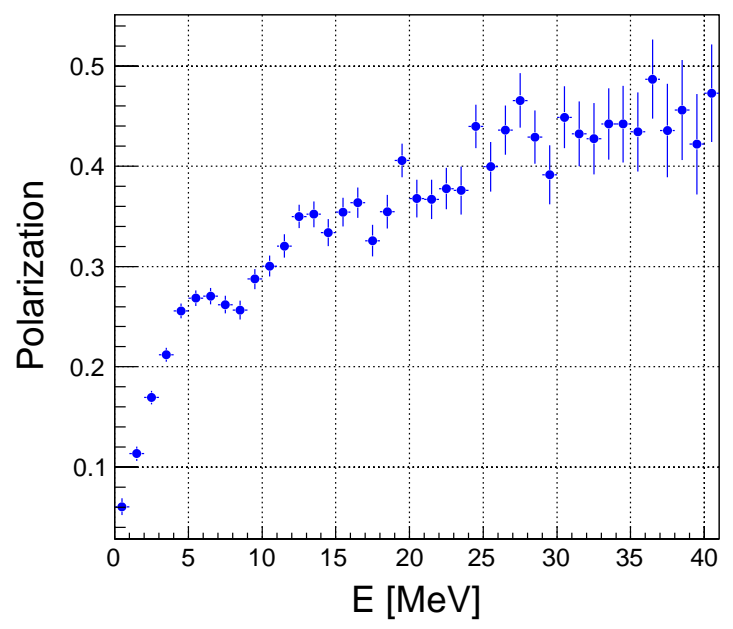

Figure 4: The positron polarization after the target.

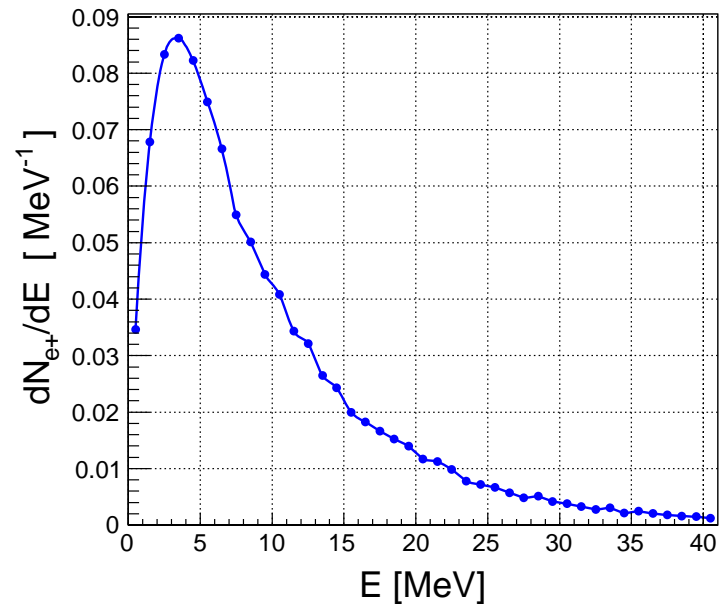

Figure 5: The positron energy distribution after the target.

\section{POSITRON CAPTURE}

Due to the very high divergence of the positron beam after the target (rms $p_{x} / p$ is 0.35 , see Fig. 2), the positron beam has to be focused in a capture section. In the positron source model considered here, the capture section consists of the adiabatic matching device (AMD) and a RF structure embedded in a focusing solenoid $[6,7,8]$. The AMD allows to match the positron beam emerging with small spot size and large divergence from the target to the acceptance of the solenoid. The AMD is a $20 \mathrm{~cm}$ length tapered solenoid starting with an initial field of $6 \mathrm{~T}$ which is reduced adiabatically down to a constant field of $0.5 \mathrm{~T}$. The AMD has a conical aperture increasing from $12 \mathrm{~mm}$ at the target side to $46 \mathrm{~mm}$ at the RF cavity side. The cavities have also $46 \mathrm{~mm}$ iris diameter. The positron capture section and preaccelerator consist of two 11-cell cavities with $14.5 \mathrm{MeV} / \mathrm{m}$ average gradient and two 17 -cell cavities with $8.5 \mathrm{MeV} / \mathrm{m}$ average gradient [9]. No space is left between target and AMD as well as AMD and accelerator cavities. More details about the simplified positron source model used for simulations could be found in [2].

The program ASTRA [10] has been used to track the positrons through the capture section. Figure 6 shows the capture efficiency (defined as ratio of captured positrons to the number of positrons after the target) versus the transverse component of momentum at the $125 \mathrm{MeV}$ position of the positron preaccelerator. The average capture efficiency is $68.4 \% .30 .3 \%$ of the positrons are lost in the AMD and the first $2 \mathrm{~m}$ of RF structures, $0.9 \%$ in the remaining part of the preaccelerator and $0.4 \%$ of positrons turn back into the target.

The captured positron beam must satisfy the dumping ring acceptance requirements. A longitudinal cut of the bunch length to $10 \mathrm{~mm}$ yields $34 \%$ reduction of the number of positrons with $1 \%$ energy spread (or $\pm 7.5^{\circ}$ of field phase). The dependence of the positron capture efficiency on the tranverse emittance cut $\epsilon_{\text {edge }}$ (diagonal edge emittance $\epsilon_{e d g e}=\epsilon_{x}+\epsilon_{y}$ ) is shown in Fig. 7. 


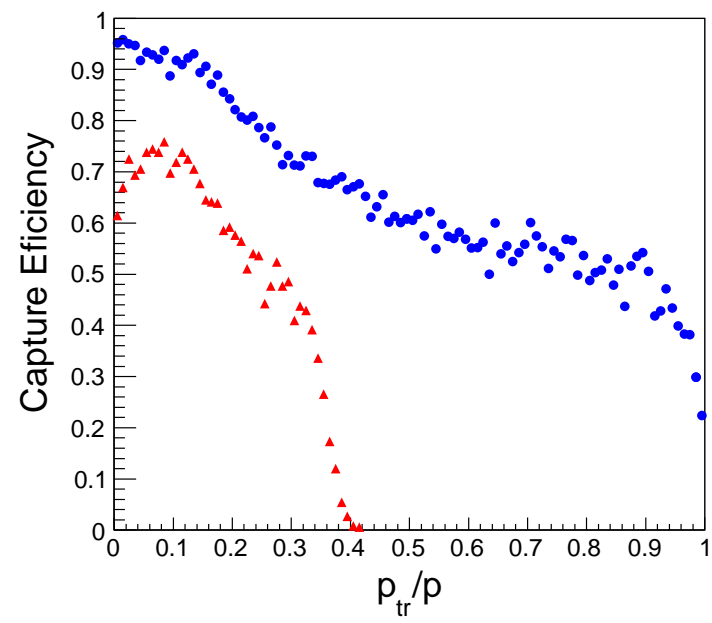

Figure 6: Positron capture efficiency vs normalized tranverse momentum $p_{t r} / p$ at the $125 \mathrm{MeV}$ point of positron preaccelerator: blue circles - without cuts; red triangles with a longitudinal bunch length cut of $10 \mathrm{~mm}$ and a tranverse diagonal emittance cut of $0.04 \pi \mathrm{rad} \mathrm{m}$.

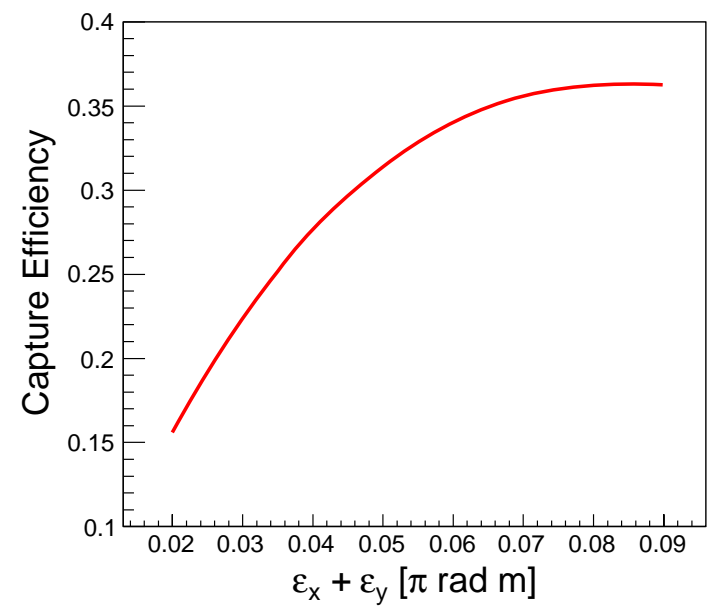

Figure 7: Positron capture efficiency vs cut on tranverse edge emittance (after applying $10 \mathrm{~mm}$ longitudinal bunch length cut) at the $125 \mathrm{MeV}$ point of positron preaccelerator.

The capture efficiency is $26.7 \%$ after applying the $10 \mathrm{~mm}$ longitudinal bunch length cut and the $0.04 \pi \mathrm{rad} \mathrm{m}$ tranverse cut (details shown in Fig. 6). The average positron polarization at $125 \mathrm{MeV}$ is $38.6 \%$ neglecting polarization degradation during the transport through the capture section.

Simulations for the W25Re target (75\% of W and $25 \%$ of $\mathrm{Re}$ ) using the same procedures as for the Ti6Al4V target give a slightly lower capture efficiency and significantly lower positron polarization. The results for both target materials are summarized in Table 1.

\section{SUMMARY}

The simulations of polarized positron production and capture have been performed. A capture efficiency of $26 \%$
Table 1: Comparison of the target materials Ti6Al4V and W25Re.

\begin{tabular}{|l|c|c|}
\hline & Ti6Al4V & W25Re \\
\hline $\mathrm{e}^{+}$yield, $\mathrm{e}^{+} / \gamma$ & $2.19 \cdot 10^{-2}$ & $3.34 \cdot 10^{-2}$ \\
\hline$E_{\text {deposited }}, \mathrm{keV} / \gamma$ & 854 & 455 \\
\hline Capture efficiency, \% & 26.7 & 23.0 \\
\hline $\mathrm{e}^{+}$polarization, \% & 38.6 & 25.1 \\
\hline
\end{tabular}

for Ti6Al4V target and a positron polarization of $38.6 \%$ has been found if an AMD is used as positron beam focusing device. The simulations have been done using FLUKA, Geant 4 and ASTRA to cover power dissipation, capture efficiency, particle tracking in the accelerator structures and polarization yield. The positron yields calculated by FLUKA and Geant 4 are in good agreement. In contrast to previous studies a helicity-dependent tracking of particles through target and capture section is possible applying the new release of Geant 4 which includes the spin dependence of all QED processes. Additional effort is necessary to achieve a detailed simulation of different positron capture devices using Geant 4 including the particle and spin tracking. This is also the focus of our ongoing work. The synergy of all three codes will allow to specify and optimize the the parameters of a polarized positron source.

\section{REFERENCES}

[1] International Linear Collider - Reference Design Report (2007), http://www.linearcollider.org.

[2] A. Ushakov, E. Elsen, K. Flöttmann, S. Riemann, and K. Sanosyan, "Radiation levels and activation at the ILC positron source", Proceedings of EPAC 2006, Edinburgh, Scotland, June 2006, p. 2478.

[3] Brian M. Kincaid, "A short-period wiggler as an improved source of synchrotron radiation”, J. Appl. Phys. 48 (1977) 2684.

[4] A. Fassò, A. Ferrari, J. Ranft, and P. R. Sala, "FLUKA: a multi-particle transport code”, CERN-2005-10 (2005).

[5] S. Agostinelli et al. [GEANT4 Collaboration], "GEANT4: A simulation toolkit", Nucl. Instrum. Meth. A 506 (2003) 250; http://geant4.web.cern.ch.

[6] H. Brechna, D. A. Hill, and B. M. Bailey, " 150 kOe liquid nitrogen cooled pulsed flux-concentrator magnet," Rev. Sci. Instrum. 36 (1965) 1529.

[7] David J. Mayhall, "A preliminary low-frequency electromagnetic analysis of a flux concentrator", UCRL-TR221994 (2006).

[8] K. Flöttmann, "Positron source option for linear colliders", EPAC'2004, Lucerne, Switzerland, 2004, p. 69.

[9] "Conceptual design of a positron pre-accelerator for the TESLA linear collider". Ed. by V. V. Paramonov and K. Flöttmann. DESY TESLA 99-14 (1999).

[10] K. Flöttmann, "ASTRA. A Space Charge Tracking Algorithm", http://www.desy.de/ mpyflo/. 